\title{
Structure-Function Correlations in Erythropoietin Formation and Oxygen Sensing in the Kidney*
}

\author{
M. Le Hir ${ }^{1}$, K.-U. Eckardt ${ }^{2,5}$, B. Kaissling ${ }^{3}$, S.T. Koury ${ }^{4}$, and A. Kurtz ${ }^{2,5}$ \\ ${ }^{1}$ Medizinische Universitätspoliklinik, Kantonsspital Basel, Institut für ${ }^{2}$ Physiologie und ${ }^{3}$ Anatomie, Universität Zürich-Irchel, \\ ${ }^{4}$ Department of Medicine, Hematology Division, Nashville, Tennessee, ${ }^{5}$ Institut für Physiologie I, Universität Regensburg
}

Summary. The kidney is the main site of erythropoietin (EPO) formation. Oxygen sensing in the kidney itself plays a major role in the control of EPO synthesis. By in situ hybridization it has been established that the EPO-producing cells are situated in the interstitium of the cortical labyrinth, but they have not been precisely identified. Morphological findings provide new insights into the location and mechanism of oxygen sensing in the kidney. In addition to causing an increase in the number of cells containing EPO messenger RNA, anemia provokes structural changes exclusively in the cortical labyrinth. Specifically, the fibroblasts become enlarged and show increased activity of 5 -nucleotidase, and the S1 segment of the proximal tubule shows similar alterations as in various models of hypoxia. Thus, structures that are situated in the close vicinity of the EPO-producing cells appear to be sensitive to decreased oxygen delivery.

Key words: Erythropoietin - Kidney - Anemia Oxygen - Proximal tubule - Endothelium - Fibroblasts

The kidney, aside from its roles in waste excretion and water and electrolyte homeostasis, carries responsibility for tissue oxygenation since it is, at least in adult life, the main site of erythropoietin (EPO) formation. EPO is a glycoprotein hormone

\footnotetext{
* Preprint of a lecture to be read at the 22nd Congress of the "Gesellschaft für Nephrologie", Heidelberg, September 1518, 1991 (Editor: Prof. Dr. E. Ritz, Heidelberg)

Abbreviations: $\mathrm{EPO}=$ erythropoietin; $\mathrm{MHC} \mathrm{II}=$ major histocompatibility class II antigen; PAS $=$ Period-acid-Schiff reaction; $\mathrm{S} 1=$ first segment of proximal tubule; $\mathrm{S} 2=$ second segment of proximal tubule
}

consisting of a polypeptide chain of 165 aminoacids and one O-linked and three $\mathrm{N}$-linked carbohydrate chains [23, 24]. It induces proliferation and differentiation of late erythroid precursors and thereby determines the rate of red cell formation [7]. EPO plasma concentrations are determined mainly by the rate of EPO production in the kidneys, which in turn is negatively controlled by oxygen delivery to the kidneys. Renal EPO formation is therefore an essential part of a negative feedback loop guaranteeing normal tissue oxygenation (Fig. 1). Thus, renal EPO production, and in consequence plasma EPO levels, are elevated during anemia (Fig. 2), arterial hypoxia, and increased oxygen affinity of hemoglobin, and they are reduced during polycythemia [3]. Circumstantial evidence, moreover, suggests that it is the renal interstitial oxygen pressure $\left(\mathrm{PO}_{2}\right)$ that governs EPO production in the kidney. The mechanism by which renal EPO formation is adapted to oxygen delivery and local $\mathrm{PO}_{2}$ is only poorly understood. In the following we will consider structural and functional relationships within the kidney that could be of importance for the oxygen-dependent regulation of EPO production.

\section{Structural Aspects of EPO Formation}

\section{Topography}

Attempts to identify the site of erythropoietin formation in the kidney have been made using both immunohistochemistry and in situ hybridization. The first method failed to reveal the EPO-producing cells because newly synthetized EPO is released into the circulation without being stored intracellularly. Thus, information about the location of EPO production within the kidney is confined to the results of in situ hybridization experiments which aimed to localize EPO messenger RNA (mRNA) 


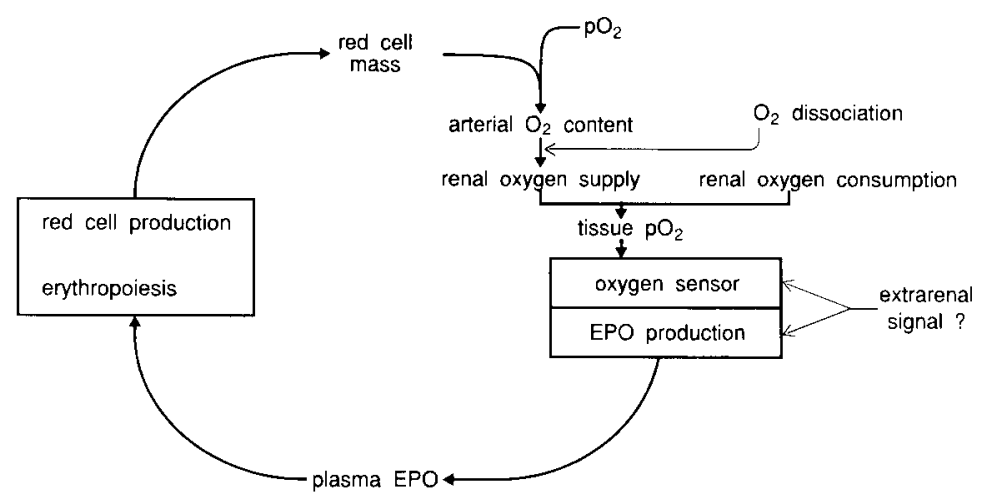

Fig. 1. Schematic representation of the feedback loop between tissue oxygenation and the production of erythropoietin

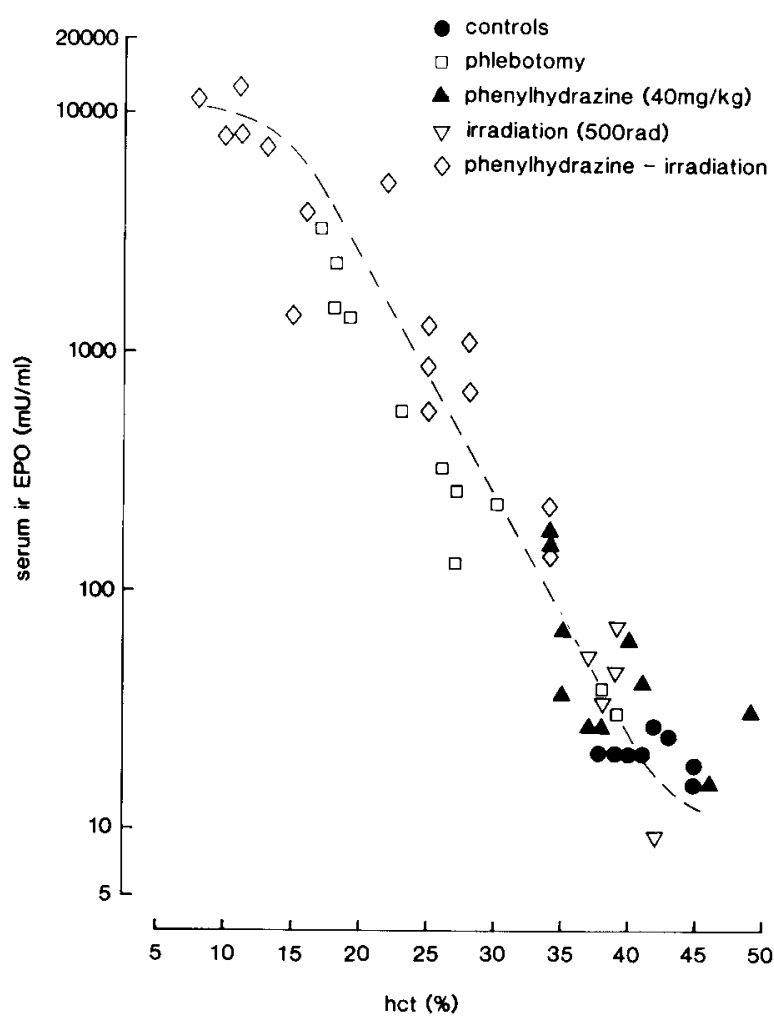

Fig. 2. Relationship between the hematocrit and the concentration of erythropoietin in serum in control rats and in anemic rats

at the cellular level. In control animals hybridization signals are found primarily in the deep region close to the cortico-medullary border [13]. The increase in the rate of EPO production in anemia is brought about not through increased synthesis by a constant cell population, but through recruitment of cells that show no synthesis under control conditions (Fig. 3). When production is maximally stimulated by profound anemia, EPO-mRNA-positive cells are homogeneously distributed through-

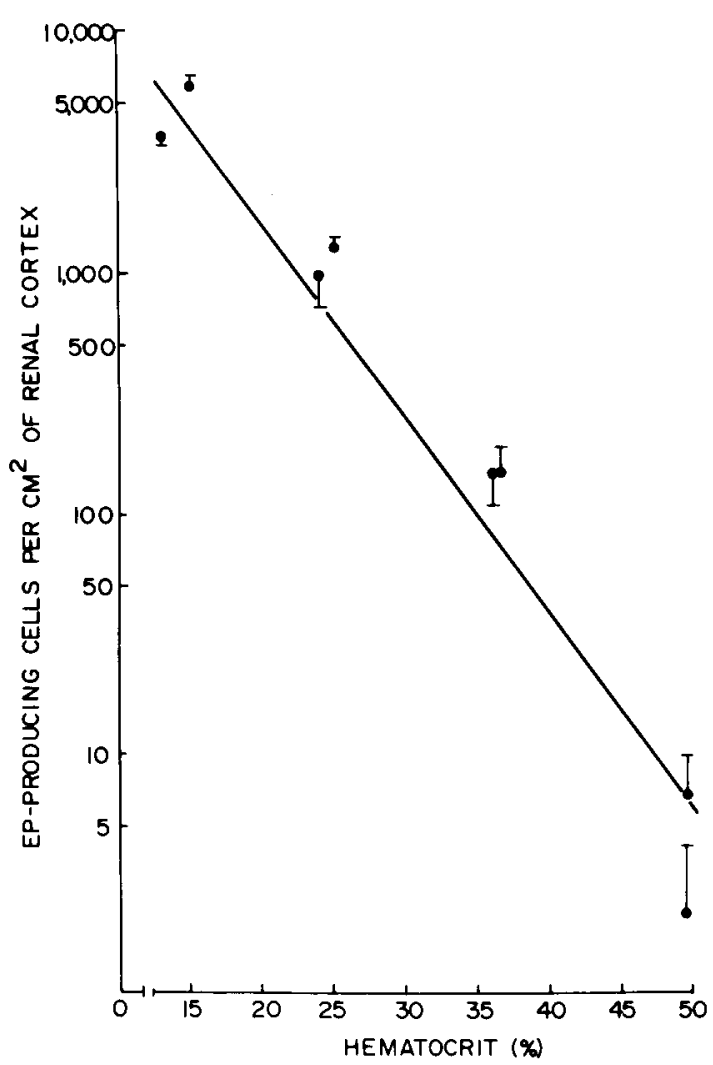

Fig. 3. Relationship between the hematocrit and the density of erythropoietin-mRNA positive cells detected by in situ hybridization in sections of the renal cortex of mice

out the cortical labyrinth, the region including the glomeruli and the large vessels (Fig. 4).

\section{Histology}

Although capillary endothelial cells have been considered the most likely EPO producers $[12,18]$, the EPO-mRNA-positive cells have not yet been identified. However, it is clear that they are present in a space that excludes glomeruli, tubules, and 

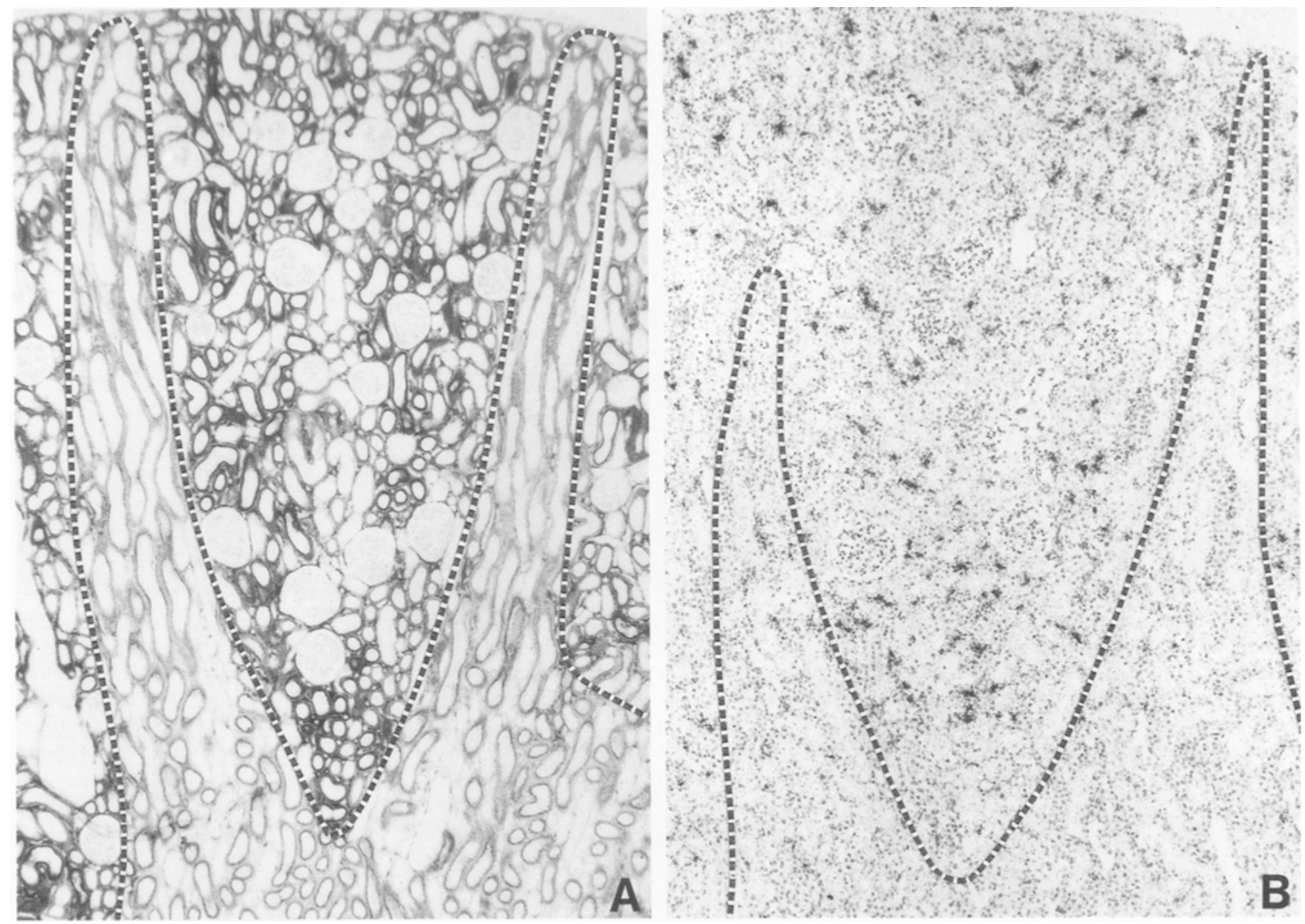

Fig. 4a, b. Topography of the production of erythropoietin in the rat kidney. Left: The cortical labyrinth is well visualized using an enzyme histochemical technique for 5'-nucleotidase, with the strong reaction seen as black deposits in the proximal convoluted tubule and in the fibroblasts. Right: In situ hybridization of erythropoietin-mRNA in the kidney of an anemic rat. The comparison of the two micrographs shows that hybridization took place in the cortical labyrinth (delimited with a dotted line), but not in the medullary rays or in the outer medulla. $\mathrm{A} \sim 62 \times, \mathrm{B} \sim 95 \times$

large vessels (Fig. 5). The cellular constituents of that space have been described in a recent review [21]. They belong to four groups: the endothelia of the peritubular capillaries, the endothelia of the lymphatics, the resident interstitial cells, and the bone marrow-derived interstitial cells. The bone marrow-derived cells, which are found throughout the kidney, have in common antigens of the major histocompatibility complex (MHC) II class [1], but they are very heteromorphic. The variety of shapes of these cells could reflect the existence of distinct cell types; accordingly, the round cells and those with cytoplasmic processes have been termed macrophages (or mononuclear cells) and dendritic cells, respectively [21]. Alternatively, one might be dealing with macrophages in the round, nonactivated state and in the stellate, activated state (monocytes and histiocytes, respectively, in the earlier literature). The resident interstitial cells consti- tute a morphologically homogeneous cell population in the cortex and the outer medulla, where they are identified by light microscopy on account of their extended cytoplasmic processes, their large size compared with the bone marrow-derived cells, the lack of MHC II antigens (unpublished observation), and, in the cortical labyrinth, the presence of the membrane enzyme ecto-5'-nucleotidase [5, 19]. They have been termed fibrocytes, fibroblastlike cells, or fibroblasts.

An interstitial location of EPO formation is further supported by examination of kidneys of rats that had developed an autoimmunity against EPO [17]. Extensive deposits of immunoglobulins were found in the extracellular matrix of the interstitium of the cortical labyrinth (Fig. 6). They might represent immune complexes that were produced when newly secreted EPO reacted with circulating antiEPO autoantibodies. 

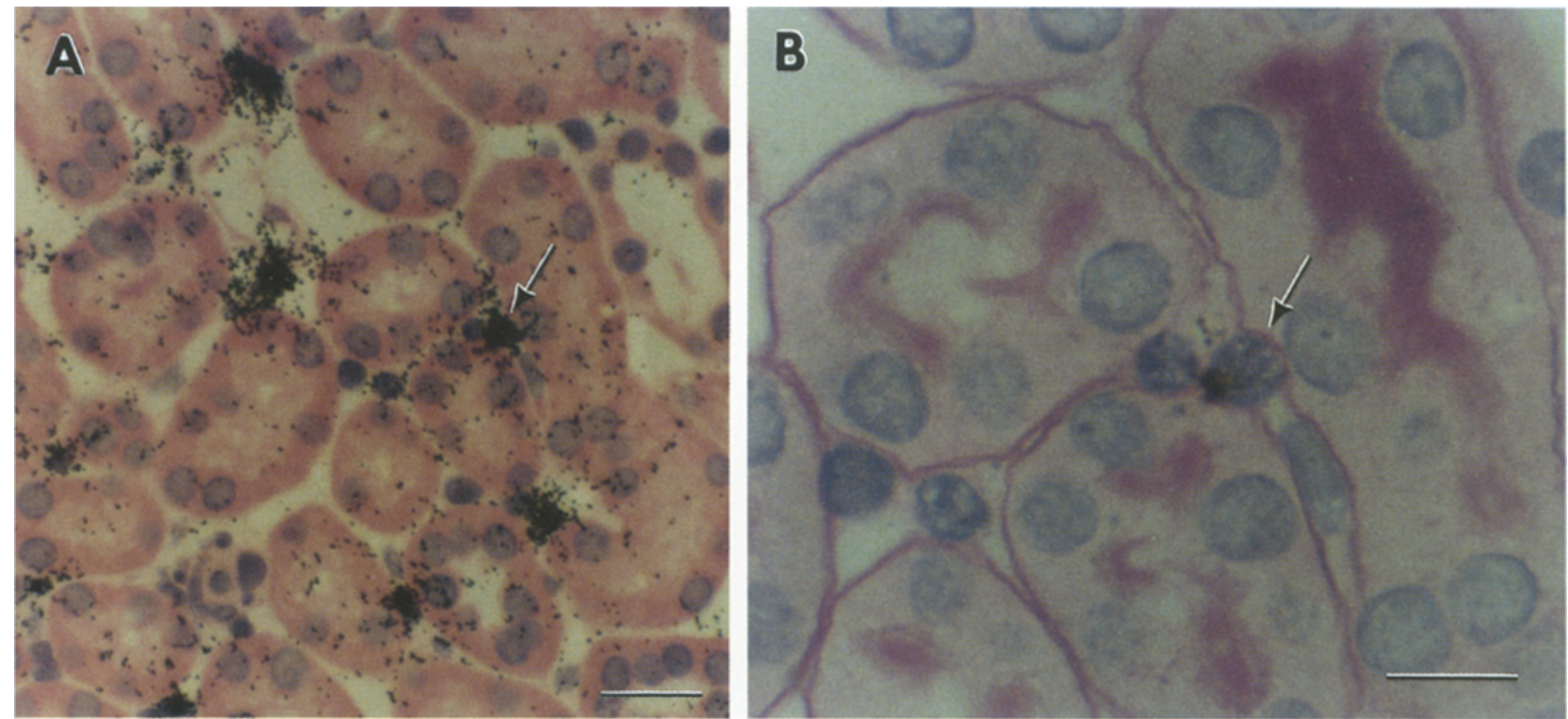

Fig. 5. Localization of erythropoietin producing cells in the mouse kidney. A Hemotoxilin and eosin stained in situ autoradiogram demonstrating the localization of EPO-producing cells in the murine renal cortex. All cells overlaid with silver grains are in the interstitium between tubules $[12,13]$. Bar $=20 \mu \mathrm{m}$. B. Higher magnification photomicrograph of an area in $\mathbf{A}$ after destaining the section with acid alcohol, removal of the silver grains from the emulsion and restaining the section using the PAS reaction [12]. The arrow points to the same cell indicated by the arrow in $\mathbf{A}$. The brush borders and basement membranes of the proximal tubules are stained in pink. Note that the EPO-producing cell lies outside the basement membranes of the proximal tubules surrounding it. $\mathrm{Bar}=10 \mu \mathrm{m}$

\section{Structural Changes in the Kidney in Response to Anemia}

Anemia, whether induced by autoimmunity against erythropoietin or by hemolysis, provoked notable histologic alterations in the rat kidney [published in part: 17, 20]. In the autoimmune model observations were made 4 weeks after immunization with large doses of human erythropoietin, when the hematocrit values ranged from $16 \%$ to $28 \%$. In the hemolytic model the rats were sacrified 5-7 days after irradiation and injection of phenylhydrazine, and the hematocrit values ranged between $8 \%$ and $15 \%$. No changes were observed in the glomeruli or in the large vessels in either model.

In the nephron the proximal convoluted tubule showed the most evident morphological effects of anemia. The alterations were distributed irregularly across the cortical labyrinth and affected mainly the S1 segment. The damage was most striking at the brush border. The microvilli became sparser or disappeared completely from extended areas of the apical cell pole. Often the cytoplasm was condensed and vacuolized and contained a few large lipid droplets. Desquamation of the epithelium was seen occasionally. The straight part of the proximal tubule, which is localized in the medullary rays and in the outer stripe of the medulla, was not affected.

The fibroblasts of anemic animals often contained lipid droplets in the cortex and in the outer medulla. In the cortical labyrinth, but not in the medullary rays or in the medulla, the fibroblasts showed further alterations $[17,20]$. They appeared enlarged, and their cytoplasmic processes became more numerous. The activity of the membrane enzyme $5^{\prime}$-nucleotidase was strongly increased (Fig. 7).

The MHC II-positive cells were not investigated in the autoimmune model of anemia. In the hemolytic model (irradiation followed by injection of the hemolytic drug phenylhydrazine), they decreased dramatically in number (unpublished results). This was probably due to irradiation, because irradiated controls that did not receive phenylhydrazine showed the same effect. Depletion of MHC II-positive cells in the kidney after irradiation has been reported by others [1]. We did not detect morphological alterations in the MHC IIpositive cells in anemia. The intensity of the labeling with anti-MHC II antibody in single cells was the same as that seen in control animals (unpublished results). 

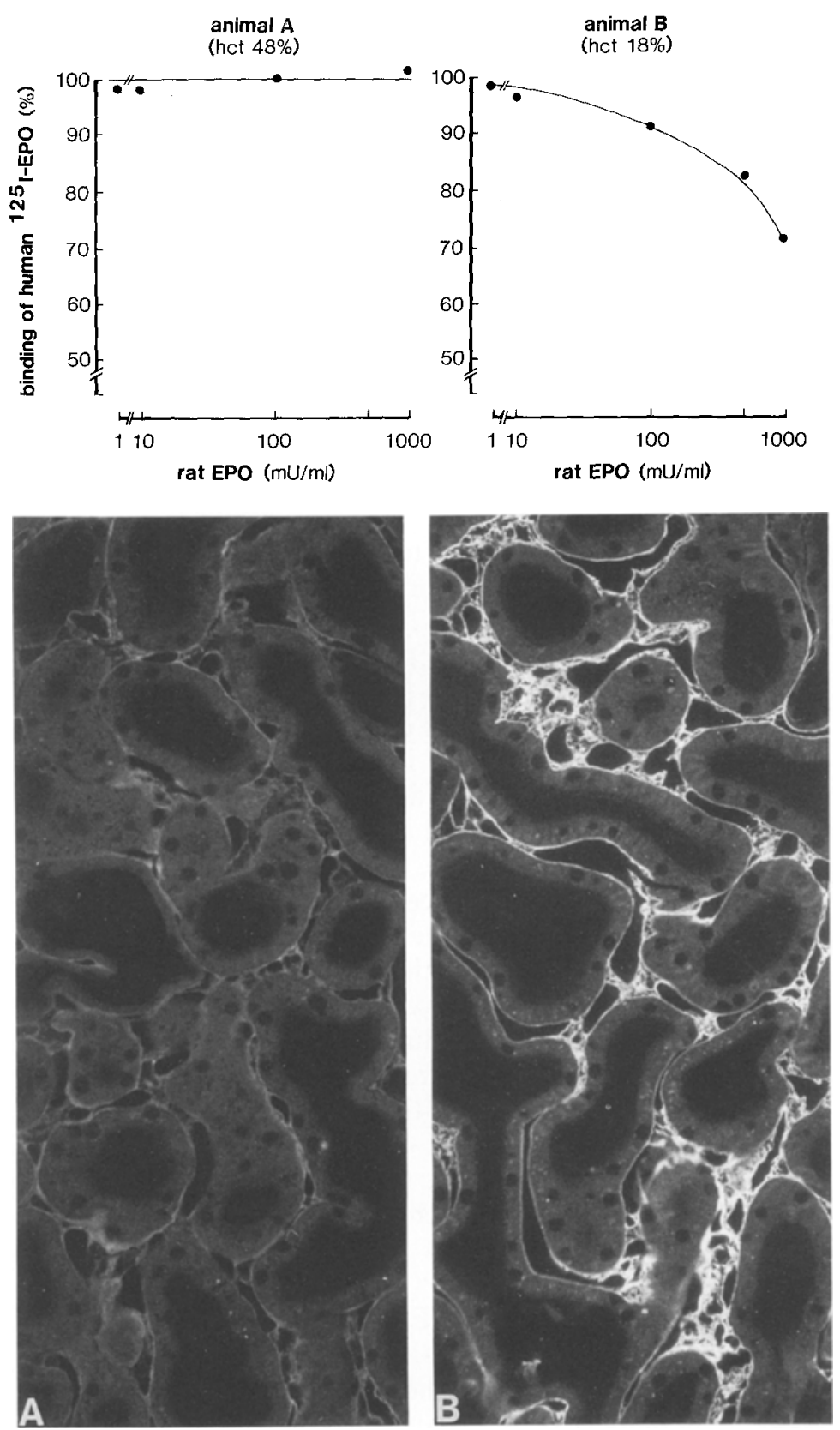

Fig. 6. Interstitial immune deposits in an autoimmune model of anemia. Rats were immunized by injection of human recombinant erythropoietin. By using a fluorescein-labeled goat anti-rat IgG antibody, rat immunoglobulins were detected in the peritubular interstitium (arrows). Left: Rat that did not develop autoimmunity and maintained a normal hematocrit. Right: Rat that produced antibodies to its own erythropoietin and therefore became anemic. Cross-reaction was tested with a radioimmunoassay (inserts at the top of the micrographs). $\sim 750 \times$

\section{Implications Concerning the Oxygen Sensor}

\section{Is the Kidney an Appropriate Site for Oxygen} Sensing?

The feedback control of erythropoiesis via EPO requires the translation of changes in blood oxygen content into specific signals that regulate EPO formation, a process commonly referred to as "oxygen sensing". Thus, renal production of EPO appears to be under the primary control of an oxygen sensor that is located in the kidney itself, although modulation by extrarenal factors may also contribute. At first glance, this hardly seems compatible with the apparently luxurious supply of oxygen to the kidney. The blood supply to this organ is very high, and the fractional extraction of oxygen amounts to only about $8 \%$. However, direct measurements of $\mathrm{PO}_{2}$ in the renal cortex yielded surprisingly low values [28]. Even the $\mathrm{PO}_{2}$ of the glomerular effluent, which was measured at the renal surface, was far below the value in the renal vein [28]. These results suggest an arterio-venous oxygen shunt. A diffusion exchange of gases between 

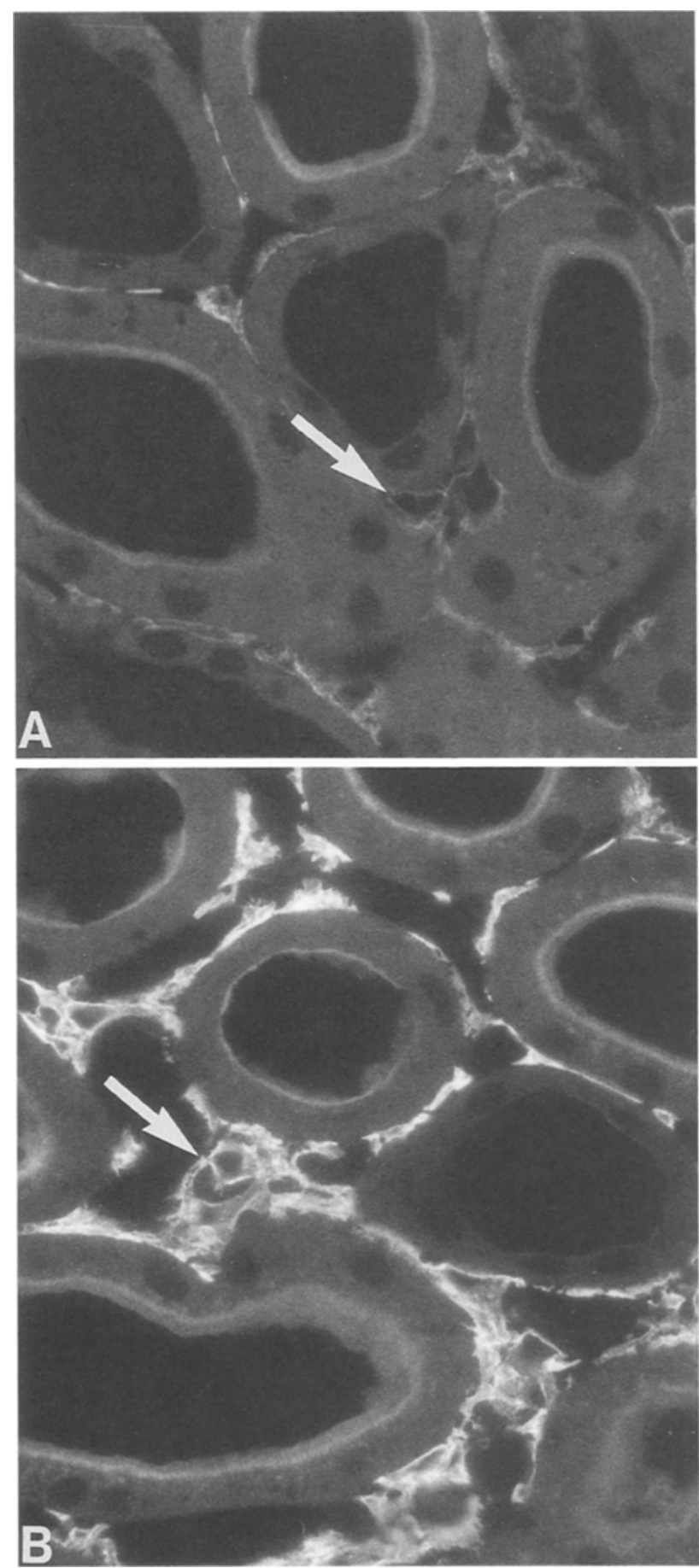

Fig. 7 a, b. Localization of $5^{\prime}$-nucleotidase in the cortical labyrinth of a control rat (A) and of an anemic rat (B). The indirect immunofluorescence technique was performed using a polyclonal anti-rat 5'-nucleotidase antiserum. Anemia provokes enlargement of the fibroblasts (arrows) and an increase in their content of $5^{\prime}$-nucleotidase. $\sim 900 \times$

arteries and veins could be possible on the basis of the intimate association of the arterial and venous trees from the entry at the renal hilus up to the surface of the cortex [16]. The well-known susceptibility of the kidney to hypoxic insults [2, 29], as exemplified above by the damage to the proximal tubule in anemic rats, constitutes a further indication that the high oxygen content in the renal vein gives a false respresentation of the oxygen supply to the parenchyma. Considering the dependency of renal function on oxidative metabolism [14], the capacity of the kidney to play the role of oxygen sensor for the regulation of production of EPO is not surprising.

\section{Role of the Proximal Tubule in Oxygen Sensing}

Since the oxygen sensor responsible for the regulation of EPO formation apparently senses the oxygen content of blood, it must involve an oxygenconsuming element. The main determinant of oxygen consumption in the kidney is the tubular reabsorption of sodium [14]. In order to identify the tubular segment, the oxygen consumption of which might be involved in "oxygen-sensing" mechanisms, site-specific diuretics were used to reduce the oxygen demand in the successive parts of the nephron [4] (Fig. 8). Substances that inhibit transport in the thick ascending limb, the distal convoluted tubule, and the collecting system did not influence the production of EPO. Participation of those segments in oxygen sensing is therefore not very likely. By exclusion one could infer that it is the oxygen consumption of the proximal tubule that is essential for the oxygen-dependent control of EPO production. This conclusion is supported by the findings that the carboanhydrase inhibitor acetazolamide, which preferentially inhibits proximal tubular sodium reabsorption, also attenuates hypoxia-induced EPO formation, and that the renal cells containing EPO mRNA are in close proximity to the proximal tubules [13].

Compared to other structures of the nephron, and also to other tissues, the proximal tubule seems to be particularly adequate to play the role of oxygen-consuming structure in "oxygen sensing". The transport work, and hence the energy consumption, of the proximal tubule is quite constant under physiological conditions, and it relies almost completely on oxygen metabolism for energy production $[11,25]$.

The role of the proximal tubule in oxygen sensing might be merely to consume oxygen, and thereby to convert a change of blood oxygen content into a change of local $\mathrm{PO}_{2}$. It might also be to generate, in response to changes in oxygen supply, specific signals that would affect the EPO-producing cells. In the first case $\mathrm{PO}_{2}$ would be directly sensed by the EPO-producing cells. The produc- 


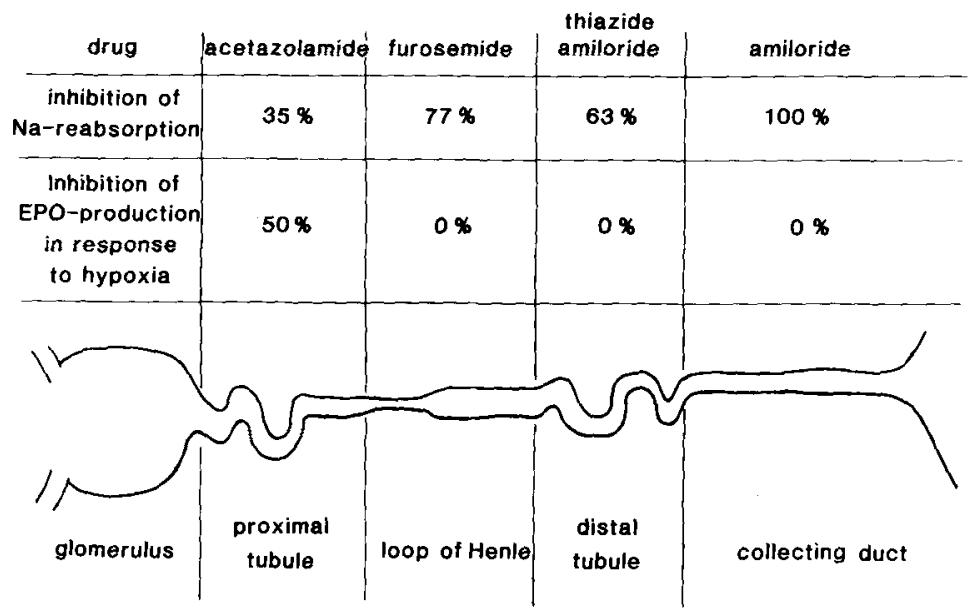

Fig. 8. Schematic representation of the effect of diuretics on the reabsorption of sodium in specific segments of the nephron and on the production of erythropoietin. Adapted from Fig. 4 tion of EPO by a hepatoma cell line in tissue culture in responses to changes of $\mathrm{PO}_{2}$ shows that a mechanism for direct oxygen sensing [6] actually exists, but there is no evidence that it operates in vivo in the kidney. The second possibility could be described as indirect oxygen sensing because the sensing of $\mathrm{PO}_{2}$ would not take place in the EPOproducing cells but in the proximal tubule. The damages provoked by ischemia, hypoperfusion, and anemia in the proximal tubule indicate that the functioning of that segment is indeed markedly sensitive to oxygen delivery $[22,29]$. This might be a consequence of the very low rate of glycolysis in the proximal tubule in comparison with other parts of the nephron $[11,25]$. A hypothetical signal released by the proximal tubule in response to an oxygen deficit would have direct access to the EPO-producing cells, the majority of which are located in close proximity to this segment [13]. The nature of such a signal and of its transduction pathway are as yet purely speculative. Nevertheless, it should be noted that in isolated rat kidneys neither stimulation of cyclic AMP and cyclic GMP formation nor application of adenosine reproduced the effect of lowering $\mathrm{PO}_{2}$ on EPO release [27].

\section{Might the Topographic Heterogeneity in EPO Production Represent Heterogeneity in Oxygen Sensing?}

The topographical heterogeneity of EPO production in the renal cortex, as revealed by in situ hybridization, might reflect differences in the capacity for oxygen sensing among different cortical regions. In normal animals renal EPO formation is found mainly in the deep cortex [13] - more precisely, in the area of the juxtamedullary glomeruli at the tips of the pyramids of the cortical labyrinth.
This might be due to a relatively poor delivery of oxygen to that region. Instead of giving rise to an extensive capillary network in the neighboring region, as in the remainder of the cortex, the efferent arterioles of juxtamedullary glomeruli descend into the medulla. Therefore, the deep regions of the cortical labyrinth rely on a fraction of the postglomerular circulation of the midcortical glomeruli for oxygen supply [16]. This specific arrangement might result in a lower oxygen delivery in that region than in more superficial parts of the cortex. Oxygen extraction is not likely to be correspondingly lower, but rather higher, in the environment of the deep glomeruli than in the remainder of the cortex. Indeed, the gomerular filtration rate, and hence the reabsorptive work, is highest in the deep nephrons [8].

Under conditions of maximal triggering of EPO formation by severe anemia, in situ hybridization reveals a homogeneous distribution of EPOmRNA-positive cells in the entire cortical labyrinth, whereas hybridization is rarely detected in the medullary rays (Fig. 4). The quasi-exclusive localization of EPO mRNA in the cortical labyrinth might be due to a higher sensitivity to alterations of blood oxygen content in that region than in the medullary rays. This proposal is supported by the following considerations. The damage to the proximal tubule during anemia is restricted to the convoluted part, which is confined to the cortical labyrinth. This can hardly be explained by intrisic differences in sensitivity to hypoxia among different parts of the proximal tubule, since in both ischemia and hypovolemic hypotension the straight part has been found to be at least as sensitive as the convoluted part $[2,29]$. In these two pathological conditions, in contrast with anemia, the transport work of the kidney is interrupted or strongly decreased. It is thus possible that the exclusive 
damage to the convoluted part in anemia reflects a higher rate of extraction of oxygen due to higher rates of active transport than in the straight part. Indeed, the activity of the Na-K-ATPase $[10,26]$ and the density of basolateral membranes and of mitochondria [16] vary markedly along the proximal tubule, suggesting large differences in active transport. These parameters are highest in the S1 segment, which is found exclusively in the labyrinth. They are intermediate in the S2 segment, which is found in the labyrinth and in the periphery of the medullary rays, and lowest in the S3 segment, which constitutes most of the straight part of the proximal tubule in the core of the medullary rays. This suggests a much higher consumption of oxygen by the proximal tubule in the cortical labyrinth than in the medullary rays. The dense packing of mitochondria at the basal half of the proximal tubular cells in S1, and to a lesser extent in S2, would be favorable to the establishment of a diffusion barrier to oxygen, which in turn might create zones of low $\mathrm{PO}_{2}$ within these cells [9].

\section{Implications Concerning the EPO-Producing Cell}

As indicated above, the distribution pattern of the EPO mRNA revealed by in situ hybridization in kidney sections limits the search for the EPO-producing cell type to four populations: fibroblasts, MHC II-positive cells, and the endothelia of lymph capillaries and blood capillaries. Lymph capillaries are found only in the periarterial tissue layers in the cortex [15]. This does not seem to correspond to the distribution of the EPO-mRNA-positive cells determined by in situ hybridization $[12,17$, $18]$.

The production of EPO can hardly be ascribed to the MHC II-positive cells, because the hemolytic model of anemia yielded the highest levels of EPO measured in rats (Fig. 2), while in that model the population of $\mathrm{MHC}$ II-positive cells was severely depleted as a consequence of irradiation (unpublished results). One could imagine that a decrease in the number of EPO-producing cells could be compensated for by an increase in the rate of production of EPO per cell. This is unlikely on the basis of observations by Koury et al. [13]. They found that the rate of EPO production is proportional to the number of producing cells (Fig. 3), as determined by in situ hybridization, and that the density of silver grains on positive cells is independent of the overall rate of production.

The fibroblasts appear to be better candidates. They constitute the main cell type in the peritubu- lar space (Fig. 7) where the EPO-producing cells have been located $[12,18]$. They obviously react to anemia, showing morphological changes as well as stimulation of the membrane enzyme 5'-nucleotidase (Fig. 7). These alterations are much more evident in the cortical labyrinth, the site of EPO production, than in the medullary rays [20]. The activity of $5^{\prime}$-nucleotidase in the fibroblasts in normal rats is highest at the tip of the pyramids of the cortical labyrinth, close to the medulla. In anemic rats the activity is homogeneously distributed across the cortical labyrinth. These patterns, under control conditions as well as in anemia, correspond to the patterns of distribution of EPO-producing cells. This congruence might be due to one and the same signal controlling both the activity of the 5 '-nucleotidase and the production of EPO. The fact that EPO production is regulated by an on/off switch would explain why the changes in the distribution of the signal for EPO mRNA with in situ hybridization is much more evident than the modifications of the gradient of $5^{\prime}$-nucleotidase between deep regions and the surface of the cortex.

A subpopulation of capillary endothelial cells was considered the most likely site of EPO production by those who carried out the first studies using in situ hybridization $[12,18]$. The localization of EPO-mRNA-positive cells is compatible with that proposal, but this is also true for the fibroblasts. No morphological effects of anemia in the renal capillary endothelium have been reported so far.

\section{References}

1. Bohman S-O, Sundelin B, Forsum U, Tribukait B (1988) Experimental depletion of different renal interstitial cell populations. Am J Med Sci 295:252-257

2. Dobyan DC, Nagle RB, Bulger RE (1977) Acute tubular necroses in the kidney following sustained hypotension. Lab Invest 37:411-422

3. Eckardt K-U, Bauer C (1989) Erythropoietin in health and disease. Eur J Clin Invest 19:117-127

4. Eckardt K-U, Kurtz A, Bauer C (1990) Regulation of erythropoietin formation is related to proximal tubular function. Am. J Physiol 256:F942-F947

5. Gandhi R, Le Hir M, Kaissling B (1990) Immunolocalization of ecto- $5^{\prime}$-nucleotidase in the kidney by a monoclonal antibody. Histochemistry 95:165-174

6. Goldberg MA, Gaut CC, Cunningham JM, Bunn HF (1987) The regulated expression of erythropoietin by two human hepatoma cell lines. Proc Natl Acad Sci USA $84: 7972-7976$

7. Graber SE, Kranz SB (1989) Erythropoietin: biology and clinical use. Hematol Oncol Clin North Am 3:369-400

8. Jacobson HR, Kokko JP (1985) Intrarenal heterogeneity: vascular and tubular. In: Sedlin DW, Giebisch $G$ (eds) The kidney: physiology and pathophysiology. Raven Press, New York, pp 531-580 
9. Jones DP, Aw TI, Sillau AH (1990) Defining the resistance to oxygen transfer in tissue hypoxia. Experientia $46: 1180$ 1185

10. Katz AI, Doucet A, Morel F (1979) Na-K-ATPase activity along the rabbit, rat and mouse nephron. Am J Physiol 237:F114-F120

11. Klein KL, Wang M-S, Torikai S, Davidson WD, Kurokawa K (1981) Substrate oxidation by isolated single nephron segments of the rat. Kidney Int 20:29-35

12. Koury ST, Bondurant MC, Koury MJ (1988) Localization of erythropoietin synthesizing cells in murine kidney by in situ hybridization. Blood 71:524-527

13. Koury ST, Koury MJ, Bondurant MC, Caro J, Graber SE (1989) Quantitation of erythropoietin-producing cell in kidneys of mice by in situ hybridization : correlation with hematocrit, renal erythropoietin mRNA, and serum erythropoietin concentration. Blood 74:645-651

14. Kramer K, Detjeen $P(1960)$ Beziehung des $\mathrm{O}_{2}$-Verbrauchs der Niere zur Durchblutung und Glomerulusfiltrat bei Änderung des arteriellen Druckes. Pfluegers Arch 271: 782 796

15. Kriz W (1987) A periarterial pathway for intrarenal distribution of renin. Kidney Int $31: \mathrm{S} 51-\mathrm{S} 56$

16. Kriz W, Kaissling B (1985) Structural organization of the mammalian kidney. In: Sedlin DW, Giebisch G (eds) The kidney: physiology and pathophysiology. Raven Press, New York, pp 265-306

17. Kurtz A, Eckardt K-U, Neumann R, Kaissling B, Le Hir $M$, Bauer C (1989) Site of erythropoietin formation. Contrib Nephrol 76:14-23

18. Lacombe C, Da Silva J-L, Bruneval P, Fournier J-G, Wendling $\mathrm{F}$, Casadevall $\mathrm{N}$, Camilleri J-P, Bariety J, Varet $\mathrm{B}$, Tambourin P (1988) Peritubular cells are the site of erythropoietin synthesis in the murine hypoxic kidney. $J$ Clin Invest $81: 620-623$

19. Le Hir, Kaissling B (1989) Distribution of 5'-nucleotidase in the renal interstitium of the rat. Cell Tissue Res 258:177182

20. Le Hir M, Eckardt K-U, Kaissling B (1989) Anemia induces $5^{\prime}$-nucleotidase in fibroblasts of cortical labyrinth of rat kidney. Renal Physiol Biochem 12:313-319
21. Lemley KV, Kriz W (1991) Anatomy of the renal interstitium. Kidney Int 39:370-382

22. Olsen S, Solez K (1987) Acute renal failure: pathogenesis in light of new morphological data. Clin Nephrol 27:271277

23. Recny M, Scoble HA, Kim Y (1987) Structural characterization of natural human urinary and recombinant DNAderived erythropoietin. J Biol Chem 262:17156-17163

24. Sasaki H, Bothner B, Dell A, Fukuda M (1987) Carbohydrate structure of erythropoietin expressed in Chinese hamster ovary cells by a human erythropoietin cDNA. J Biol Chem 262:12059-12076

25. Schmid H, Mall A, Scholz M, Schmidt U (1980) Unchanged glycolytic capacity in rat kidney under conditions of stimulated gluconeogenesis. Determination of phosphofructokinase and pyruvate kinase in microdissected segments of fasted and acidotic animals. Hoppe Seyler's Z Physiol Chem $361: 819-829$

26. Schmidt U, Dubach UC (1969) Activity of $\left(\mathrm{Na}^{+}, \mathrm{K}^{+}\right)$-stimulated adenosine triphosphatase in the rat nephron. Pfluegers Arch 306:219-226

27. Scholz H, Schurek HJ, Eckardt K-U, Kurtz A, Bauer C (in press) Oxygen dependent erythropoietin production by the isolated perfused rat kidney. Pfluegers Arch

28. Schurek HJ, Jost U, Baumgärtl H, Bertram H, Heckmann U (1990) Evidence for a preglomerular oxygen diffusion shunt in rat renal cortex. Am J Physiol 259:F910-F915

29. Venkatachalam MA, Bernard DB, Donohoe JF, Levinsky NG (1978) Ischemic damage and repair in the rat proximal tubule: differences among the S1, S2 and S3 segments. Kidney Int 14:31-49

Received: April 1, 1991

Accepted: May 11, 1991

Prof. Dr. B. Kaissling

Universität Zürich-Irchel

Anatomisches Institut

Winterthurerstr. 190

CH-8057 Zürich, Schweiz 